\title{
ANOTHER CLASS OF INVERTIBLE OPERATORS WITHOUT SQUARE ROOTS
}

\author{
DON DECKARD AND CARL PEARCY
}

1. Introduction. In [2], Halmos, Lumer, and Schäffer exhibited a class of invertible operators on Hilbert space possessing no (bounded) operator square roots, thereby settling what was until then an open question. This class of examples was obtained by considering the Hilbert space of all analytic functions which are square-integrable (with respect to planar Lebesgue measure) on a bounded domain $D$ in the complex plane. In this setting they show that the "analytic position operator" defined by $(A f)(t)=t f(t)$ has a square root if and only if $D$ contains no Jordan curve surrounding zero.

The present authors, while trying to discover the limitations of Corollary 4.2 [1], were led to a different, matrix-theoretic example of an invertible operator on Hilbert space which has no (bounded) operator square root. In fact considerations of spectra show that the class of examples exhibited here is disjoint from the class appearing in [2].

2. An example. The following considerations lead to the construction of the above mentioned example. Let $N=\left\{n_{k}\right\}_{k=1}^{\infty}$ be any strictly increasing sequence of positive integers such that $n_{1} \geqq 3$. For simplicity of notation in what follows, we shall write $n \in N$ to mean "for some positive integer $k, n=n_{k}$ " and $\left\{a_{n}\right\}_{n \in N}$ or simply $\left\{a_{n}\right\}$ instead of $\left\{a_{n_{k}}\right\}_{k=1}^{\infty}$. Let $\mathfrak{F C}_{n}$ be $n$-dimensional Hilbert space, and let $M_{n}$ be the algebra of all operators on $\mathfrak{K C}_{n}$. Denote by $\mathfrak{F C}$ the Hilbert space $\sum_{n \in N} \oplus \mathcal{H C}_{n}$, and by $R$ the $W^{*}$-algebra $\sum_{n \in N} \oplus M_{n}$. Then of course $R$ consists of all operators $A=\sum \oplus A_{n}$ where $A_{n} \in M_{n}$ and the sequence $\left\{\left\|A_{n}\right\|\right\}$ is bounded. The operators which we shall consider can most easily be described by setting forth matrices, so we assume given for each $n \in N$ an orthonormal basis $X_{n}$ of $\mathfrak{H C}_{n}$, and the matrices exhibited are to be regarded as the corresponding operators. We now define the class $\Gamma \subset R$ of operators as follows: $T=\sum \oplus T_{n} \in \Gamma$ if and only if for each $n \in N, T_{n}$ has the form

$$
\left(\begin{array}{ccccc}
\omega_{n}^{1} & s_{n} & & & f_{n} \\
& \omega_{n}^{2} & s_{n} & 0 \\
& & \ddots & \ddots \\
& & \ddots & s_{n} \\
& & & \omega_{n}^{n}
\end{array}\right),
$$

Received by the editors February 15, 1962 and, in revised form, March 23, 1962. 
where

(1) for $1 \leqq r \leqq n, \omega_{n}^{r}=e^{i 2 \pi r / n}$,

(2) the sequence $\left\{s_{n}\right\}_{n \in N}$ satisfies $0 \leqq\left|s_{n}\right|<\delta$ for some $\delta<1$ and $\lim _{n \in N} n\left|s_{n}\right|=\infty$.

(3) the sequence $\left\{\left|f_{n}\right|\right\}$ is bounded.

The inverse of each $T_{n}$ satisfying $\left(^{*}\right)$ can be computed directly, and an easy calculation shows that $\left\|T_{n}^{-1}\right\| \leqq\left(1-\left|s_{n}\right|\right)^{-1}+\left|f_{n}\right|$. It follows from (2) and (3) that each $T \in \Gamma$ is invertible in $R$, and the following lemmas enable us to determine a subclass of $\Gamma$ consisting of operators having no square roots in $R$.

Lemma 2.1. Suppose that $n>1$ and that for $1 \leqq r \leqq n, \lambda_{n}^{r}$ are complex numbers satisfying $\left(\lambda_{n}^{r}\right)^{2}=\omega_{n}^{r}$. Then

$$
\lambda_{n}^{r}= \pm e^{i \pi r / n}
$$

and either

( $\alpha$ ) for some $r$ satisfying $1 \leqq r \leqq n-1,\left|\lambda_{n}^{r}+\lambda_{n}^{r+1}\right|<\pi / n$, or

( $\beta$ ) the sign in (**) is the same for $1 \leqq r \leqq n$, in which case $\left|\lambda_{n}^{1}+\lambda_{n}^{n}\right|$ $<\pi / n$.

The proof is trivial and is omitted.

Lemma 2.2. Suppose that $K$ is an operator on a Hilbert space $\mathfrak{H C}$ having the distinct eigenvalues $\left\{\lambda_{i}\right\}_{i \in I}$, and suppose that for $i \in I$, the eigenspace corresponding to $\lambda_{i}$ is spanned by the vector $x_{i}$. If $J$ is any operator on $\mathfrak{H C}$ such that $J^{2}=K$, and $\mathscr{L}$ is any subspace of $\mathfrak{H C}$ spanned by some subset of the $\left\{x_{i}\right\}_{i \in I}$, then $\mathcal{L}$ is an invariant subspace for $J$.

Proof. It suffices to show that for $i \in I, J x_{i}=\alpha_{i} x_{i}$ for some scalar $\alpha_{i}$. If $J x_{i}=y_{i}$, then $K y_{i}=K J x_{i}=J K x_{i}=\lambda_{i} y_{i}$, so that by hypothesis $y_{i}=\alpha_{i} x_{i}$.

We obtain as immediate corollaries

COROLlaRy 2.3. If $K$ is an $n \times n$ complex matrix in upper triangular form and having $n$ distinct eigenvalues, and $J$ is any square root of $K$, then $J$ is also in upper triangular form.

Corollary 2.4. Suppose $K \in R$ satisfies the hypotheses of Lemma 2.2 and the additional hypothesis that the vectors $\left\{x_{i}\right\}_{i \in I}$ span $\mathcal{H}$. Then every (bounded) square root of $K$ on $\mathcal{H}$ is in $R$.

For each sequence $\sigma=\left\{s_{n}\right\}_{n \in N}$ satisfying (2) of $\left(^{*}\right)$, consider the operator $P_{\sigma}=\sum \oplus P_{n} \in \Gamma$ defined by taking the sequence $\left\{f_{n}\right\}$ to be identically zero. An induction argument similar to the one carried 
out in $\$ 4$ of [1] yields the following fact: for each $n \in N$, there is exactly one matrix $Q_{n}$ of the form

$$
\left(\begin{array}{cccc}
\omega_{2 n}^{1} & & & \\
& 2 & & q_{i j}^{(n)} \\
& \omega_{2 n} & & \\
& & \ddots & \\
& 0 & & \cdot \begin{array}{c}
n \\
\omega_{2 n}
\end{array}
\end{array}\right)
$$

such that $Q_{n}^{2}=P_{n}$. Thus the numbers $q_{i j}^{(n)}$ are uniquely determined, and for $n \in N$ we define the sequence $\left\{\begin{array}{c}c_{n}^{\sigma} \\ \}\end{array}\right.$ by $c_{n}^{\sigma}=\sum_{j=2}^{n-1} q_{1 j}^{(n)} q_{j n}^{(n)}$.

Lemma 2.5. If $\sigma=\left\{s_{n}\right\}_{n \in N}$ satisfies (2) of $\left(^{*}\right)$, and the operator $A_{\sigma}=\sum \oplus A_{n} \in \Gamma$ is defined by setting the sequence $\left\{f_{n}^{\sigma}\right\}$ equal to $\left\{1+c_{n}^{\sigma}\right\}$ if $\left\{\left|c_{n}^{\sigma}\right|\right\}$ is bounded and equal to the null sequence if $\left\{\left|c_{n}^{\sigma}\right|\right\}$ is unbounded, then $A_{\sigma}$ has no square root in $R$.

Proof. Suppose, to the contrary, that $A_{\sigma}$ has a square root $B=\sum \oplus B_{n} \in R$. Then for $n \in N, B_{n}^{2}=A_{n}$, and it results from Corollary 2.3 that each $B_{n}$ is in upper triangular form-say

$$
B_{n}=\left(\begin{array}{ccccc}
\lambda_{n}^{1} & & & \\
& \lambda_{n}^{2} & & b_{i j}^{(n)} \\
& & \ddots & \\
& & \ddots & \\
& & & \ddots & \lambda_{n}^{n}
\end{array}\right)
$$

Clearly we can assume without loss of generality that $\lambda_{n}^{1}=\omega_{2 n}^{1}$ for each $n \in N$. A trivial calculation yields $b_{i, i+1}^{(n)}=s_{n} /\left(\lambda_{n}^{i}+\lambda_{n}^{i+1}\right)$ for $1 \leqq i \leqq n-1$ and for all $n \in N$. Since the sequence $\left\{\left\|B_{n}\right\|\right\}$ is bounded, and since $\left\{s_{n}\right\}$ satisfies (2) of $\left({ }^{*}\right)$, it follows from Lemma 2.1 that there is a positive integer $n_{0}$ such that for all $n>n_{0}$ and for $1 \leqq i \leqq n$, $\lambda_{n}^{i}=\omega_{2 n}^{i}$. Furthermore, since the matrices $A_{n}$ and $P_{n}$ always agree except for the $(1, n)$ th entry, and since for $n>n_{0}$ the principal diagonals of the matrices $Q_{n}$ and $B_{n}$ agree, it is easy to see that for $n>n_{0}$ the matrices $Q_{n}$ and $B_{n}$ must also agree except for the $(1, n)$ th entry. Thus for $n>n_{0}, b_{1 n}^{(n)}=\left(f_{n}^{\sigma}-c_{n}^{\sigma}\right) /\left(\lambda_{n}^{1}+\lambda_{n}^{n}\right)$, and since $\left|\lambda_{n}^{1}+\lambda_{n}^{n}\right|$ $<\pi / n$ for such $n$, we know by virtue of the way the sequence $\left\{f_{n}^{\sigma}\right\}$ was constructed that the sequence $\left\{\left|b_{1 n}^{(n)}\right|\right\}$ is unbounded, contradicting the assumption that the sequence $\left\{\left\|B_{n}\right\|\right\}$ is bounded. 
3. We are now in a position to prove the

TheOREM. There is an invertible operator $B$ on any Hilbert space JC such that $B$ has no square root in the algebra of all bounded operators on $\mathcal{H}$.

Proof. It is easy to see (in view of Lemma 2.2) that it suffices to prove the theorem in the case that $\mathcal{H}$ is separable. Let $A_{\sigma}=\sum \oplus A_{n}$ be as in Lemma 2.5, and define the operator $B \in R$ by $B=$ $\sum \oplus(1+1 / n) A_{n}$. Since $A_{\sigma}$ is an invertible operator on $\mathcal{H C}$, so is $B$, and by virtue of Corollary 2.4, any (bounded) square root of $B$ must be an operator in $R$. But $B$ cannot have a square root in $R$, since $A_{\sigma}$ does not, and thus the proof is complete.

As another easy corollary of Lemma 2.5 , we obtain

Corollary 3.1. If $S$ is any finite $W^{*}$-algebra of type $I$ possessing $n_{k}$-homogeneous summands with $n_{k} \rightarrow \infty$, then there is an invertible element $A \in S$ such that $A$ has no square root in $S$.

Proof. Any $W^{*}$-algebra $S$ which satisfies the hypothesis of the theorem can be written as a direct sum $S=\sum_{n \in N} \oplus S_{n}$ where $N$ is an unbounded set of positive integers and each $S_{n}$ is an $n$-homogeneous $W^{*}$-algebra [4]. If $\mathfrak{X}_{n}$ is taken to be the maximal ideal space of the center of $S_{n}$, then (see [5]) $S$ is $C^{*}$-isomorphic to the algebra $S_{0}$ which is the $C^{*}$-sum of the homogeneous $W^{*}$-algebras $\left\{M_{n}\left(\mathfrak{X}_{n}\right)\right\}_{n \in N}$ where the $M_{n}\left(\mathfrak{X}_{n}\right)$ are as defined in [1]. Thus it suffices to prove the theorem in $S_{0}$, and an invertible element $G=\left\{G_{n}\right\} \in S_{0}$ having no square root in $S_{0}$ can be obtained by choosing $A_{\sigma}=\sum \oplus A_{n}$ as in Lemma 2.5 and then defining $G_{n}(t) \equiv A_{n}$ for $t \in \mathfrak{X}_{n}$ and $n \in N$. If $G$ had a square root $J=\left\{J_{n}\right\} \in S_{0}$, then choosing arbitrary points $t_{n} \in \mathfrak{X}_{n}$ for $n \in N$, one would have $\left[J_{n}\left(t_{n}\right)\right]^{2}=G_{n}\left(t_{n}\right)=A_{n}$, contradicting the fact that $A_{\sigma}$ has no square root in $R$. Thus the proof is complete.

\section{Remarks.}

(1) Hille and Phillips have shown ([3], Theorem 9.5.4) that a sufficient condition for an operator $A$ to have roots of all orders in the Banach algebra generated by $A$ and the identity is that zero belong to the unbounded component of the resolvent set of $A$.

(2) Each analytic position operator appearing in [2] is such that the compression spectrum of the operator (in the terminology of [2]) contains an open set. On the other hand, it is clear that the operator $B$ without square root exhibited above has discrete compression spectrum, so that $B$ cannot be an analytic position operator. 
(3) If $N$ contains only odd integers, it is not hard to see that the operator $B$ appearing in the proof of the Theorem is such that $B^{2}$ has no bounded fourth root. This shows that invertible operators can have roots of some order without having roots of all orders.

\section{REFERENCES}

1. D. Deckard and C. Pearcy, On matrices over the ring of continuous complexvalued functions on a Stonian space, Proc. Amer. Math. Soc. (to appear).

2. P. R. Halmos, G. Lumer, and J. Schäffer, Square roots of operators, Proc. Amer. Math. Soc. 4 (1953), 142-149.

3. E. Hille and R. S. Phillips, Functional analysis and semigroups, Amer. Math. Soc. Colloq. Publ. Vol. 31, Amer. Math Soc., Providence, R. I., 1957.

4. I. Kaplansky, Algebras of type I, Ann. of Math. (2) 56 (1952), 460-472.

5. C. Pearcy, A complete set of unitary invariants for operators generating finite $W^{*}$-algebras of type I, Pacific J. Math. (to appear).

RICE UNIVERSITY AND

Humble OIl and Refining Company 